\title{
Bilirubin adsorption versus plasma exchange for hyperbilirubinemia in patients after cardiac surgery: a retrospective study
}

Ke Pan ${ }^{1 \dagger}$, He Zhang ${ }^{2 \dagger}$, Kai Zhong ${ }^{3}$, Hai-tao Zhang ${ }^{2}$, Ze-shi Li ${ }^{2}$, Zhong Chen ${ }^{3}$, Su-ping Gu ${ }^{4}$, Man Xie ${ }^{4}$, Tuo Pann ${ }^{2,4}$, Hai-long Cao ${ }^{4^{*}}$ and Dong-jin Wang ${ }^{1,2,3,4^{*}}$ (D)

\begin{abstract}
Objective: Hyperbilirubinemia after cardiac surgery increases in-hospital mortality and is associated with poor prognosis. Our present study aimed to compare the efficacy of bilirubin adsorption (BA) and plasma exchange (PEX) in patients with hyperbilirubinemia after cardiac surgery.
\end{abstract}

Methods: We retrospectively included patients who underwent BA treatment or PEX treatment due to severe hyperbilirubinemia after cardiac surgery at our center from 2015 to 2020. We collected results from urine and liver function tests before and after treatment and compared the in-hospital mortality and morbidity between the two treatment groups.

Results: A total of 56 patients were enrolled in this study: 14 patients received BA treatment, and 42 patients received PEX treatment. Compared to the PEX group, the BA group exhibited a statistically significant reduction in total bilirubin $(p=0.016)$ and direct bilirubin $(p=0.036)$ levels. The in-hospital mortality was $85.7 \%(48 / 56)$ in the whole group, and the BA group had a lower mortality than the PEX group ( $71.4 \% \mathrm{vs.} 90.5 \%, p=0.078)$. The BA group showed better circulatory support, including lower risks of IABP ( $21.4 \%$ vs. $52.4 \%, p=0.044)$, ECMO (21.4\% vs. 50.0\%, $p=0.061)$, reintubation ( $64.3 \%$ vs. $40.5 \%, p=0.122)$ and ventricular arrhythmias $(64.3 \%$ vs. $45.2 \%, p=0.217)$. The in-hospital mortality was still lower in the BA treatment group than in the PEX treatment group $(71.4 \%$ vs. $100 \%$, $p=0.049$ ) in the matched cohort.

Conclusions: Compared to PEX treatment, BA treatment had a higher bilirubin removal ability in patients with hyperbilirubinemia and could reduce the mortality and risks of poor clinical outcomes. BA treatment should be considered an effective treatment method for patients with higher total bilirubin or direct bilirubin levels.

Keywords: Hyperbilirubinemia, Bilirubin adsorption, Plasma exchange, Cardiac surgery

\footnotetext{
*Correspondence: 13675186233@163.com; dongjin_wang@126.com

${ }^{\dagger} \mathrm{Ke}$ Pan and He Zhang contributed equally to this work

${ }^{1}$ Department of Cardio-Thoracic Surgery, Nanjing Drum Tower Clinical

College of Xuzhou Medical University, Xuzhou, China

${ }^{4}$ Department of Cardio-Thoracic Surgery, Nanjing Drum Tower Hospital,

The Affiliated Hospital of Nanjing University Medical School, Number 321

Zhongshan Road, Jiangsu 210008, China
}

Full list of author information is available at the end of the article

(c) The Author(s) 2021. Open Access This article is licensed under a Creative Commons Attribution 4.0 International License, which permits use, sharing, adaptation, distribution and reproduction in any medium or format, as long as you give appropriate credit to the original author(s) and the source, provide a link to the Creative Commons licence, and indicate if changes were made. The images or other third party material in this article are included in the article's Creative Commons licence, unless indicated otherwise in a credit line to the material. If material is not included in the article's Creative Commons licence and your intended use is not permitted by statutory regulation or exceeds the permitted use, you will need to obtain permission directly from the copyright holder. To view a copy of this licence, visit http://creativecommons.org/licenses/by/4.0/. The Creative Commons Public Domain Dedication waiver (http://creativeco mmons.org/publicdomain/zero/1.0/) applies to the data made available in this article, unless otherwise stated in a credit line to the data. 


\section{Introduction}

Cardiac surgery with cardiopulmonary bypass (CPB) can lead to different degrees of liver damage. In a previous series of reports, the rate of hyperbilirubinemia was reported to be approximately $8.6 \%$ to $40 \%$ [1-3]. Hyperbilirubinemia significantly increases the risk of mortality and morbidity [4]. The most serious level of liver damage is acute liver failure (ALF). ALF usually develops into multiple organ dysfunction syndrome (MODS) after cardiac surgery. The rate of MODS combined with ALF is relatively low (4.7\%), while it could increase the mortality rate up to $90 \%[5,6]$.

Current treatments for ALF or hyperbilirubinemia include plasma exchange (PEX), molecular adsorbent recirculating system (MARS), extracorporeal blood purification and bilirubin adsorption (BA). All of these treatment methods are based on their ability to remove endotoxin, cytokines and bilirubinemia from blood, thereby functioning in detoxification and creating conditions for liver cell regeneration.

PEX is a nonbiological artificial liver support system and has been an effective method for treating ALF. PEX treatment is recommended for the early stage of viral hepatitis and liver failure. BA is an important part of the artificial liver support system to overcome adverse reactions such as plasma dosage restrictions, plasma allergies and blood transfusion infection. It is another option for the treatment of ALF and hyperbilirubinemia.

Multiple processes are associated with ALF in patients after cardiac surgery, including $\mathrm{CPB}$, low cardiac output syndrome and elevation of venous pressure. Therefore, it remains controversial which treatment for ALF after cardiac surgery has better clinical outcomes. Furthermore, only a few clinical studies with limited sample sizes have been performed to address this question. PEX and BA have been used at our center for the treatment of ALF after cardiac surgery since 2015 . We designed this retrospective study to compare the effectiveness of BA and PEX treatment in patients with ALF after cardiac surgery.

\section{Methods \\ Study population}

From 2015 to 2020, 11,483 adult patients underwent cardiac surgery at our center, 56 of whom were diagnosed with AFL or hyperbilirubinemia after cardiac surgery. Fourteen patients who received BA treatment were chosen from 2019 to 2020, and forty-two patients who received PEX treatment were chosen from 2015 to 2020. This study was approved by the Medical Ethics Committee of Affiliated Nanjing Drum Tower Hospital, Nanjing University Medical College (2020-249-01). The requirement for informed consent from the patients was waived, and all the authors had full control of the data and information regarding this study.

\section{Indications}

We diagnosed ALF based on the following criteria [7-9]: lab indication of jaundice progressing quickly with total bilirubin (TBil) $\geq 10 \times$ upper limit of normal (ULN) or daily increasing data $\geq 17.1 \mu \mathrm{mol} / \mathrm{L}$. Patients with ALF usually have hyperbilirubinemia and develop multiple organ dysfunction (MODS) after cardiac surgery. We divided the 56 patients into two groups: 14 patients who received BA treatment and 42 who received PEX treatment.

\section{Treatment approach}

Vascular access was obtained via a double-lumen hemodialysis catheter introduced into the femoral, jugular or subclavian veins. Blood anticoagulation was controlled using unfractionated heparin (target clotting time of 140-200 s).

For the BA treatment device, PF 2000N (Gambro Dialysatoren $\mathrm{GmbH}$ ) was used as the plasma filter, BS330 (Jafron Biomedical Co., Ltd, China) as the bilirubin absorption column (Jafron Biomedical Co., Ltd, China), HA330-II (Jafron, China) as the disposable hemoperfusion cartridge, and the Diapact CRRT system (Fresenius Medical Care, Germany) to perform the procedure; the following parameters were applied: blood flow rate 120 $150 \mathrm{ml} / \mathrm{min}$; plasma flow rate $30 \mathrm{ml} / \mathrm{min}$; average duration of treatment $4 \mathrm{~h}$; and average amount of plasma $6 \mathrm{~L}$.

For the PEX treatment device, PF 2000N (Gambro Dialysatoren $\mathrm{GmbH}$ ) was used as the plasma filter, and the Diapact CRRT system (Fresenius Medical Care, Germany) was used to perform the procedure; the following parameters were applied: blood flow rate $120-150 \mathrm{ml} /$ min; plasma flow rate $25 \mathrm{ml} / \mathrm{min}$; average duration of treatment $2 \mathrm{~h}$; and average amount of plasma $3 \mathrm{~L}$.

\section{Statistical analysis}

Data analysis was performed using SPSS for Windows version 22 (IBM Corporation, Armonk, NY). Generally, continuous variables are presented as the mean \pm standard deviation. Categorical variables are presented as absolute numbers and proportions (n, \%). Differences in categorical variables were analyzed using the $\chi^{2}$ test. Differences in continuous variables were tested by $\mathrm{t}$-test or the Mann-Whitney U-test. Some bias might exist in our study. Adjustment for indication bias was further assessed using a matched cohort. To overcome the bias in our study, we used a matched cohort of 28 patients (14 who received BA treatment and 14 who received PE treatment) with similar conditions. In the matched cohort, the PEX subjects were selected and matched for sex, age ( \pm 2 years) and EuroSCORE II $( \pm 1 \%)$. Differences were considered significant at $\mathrm{p}<0.05$. 


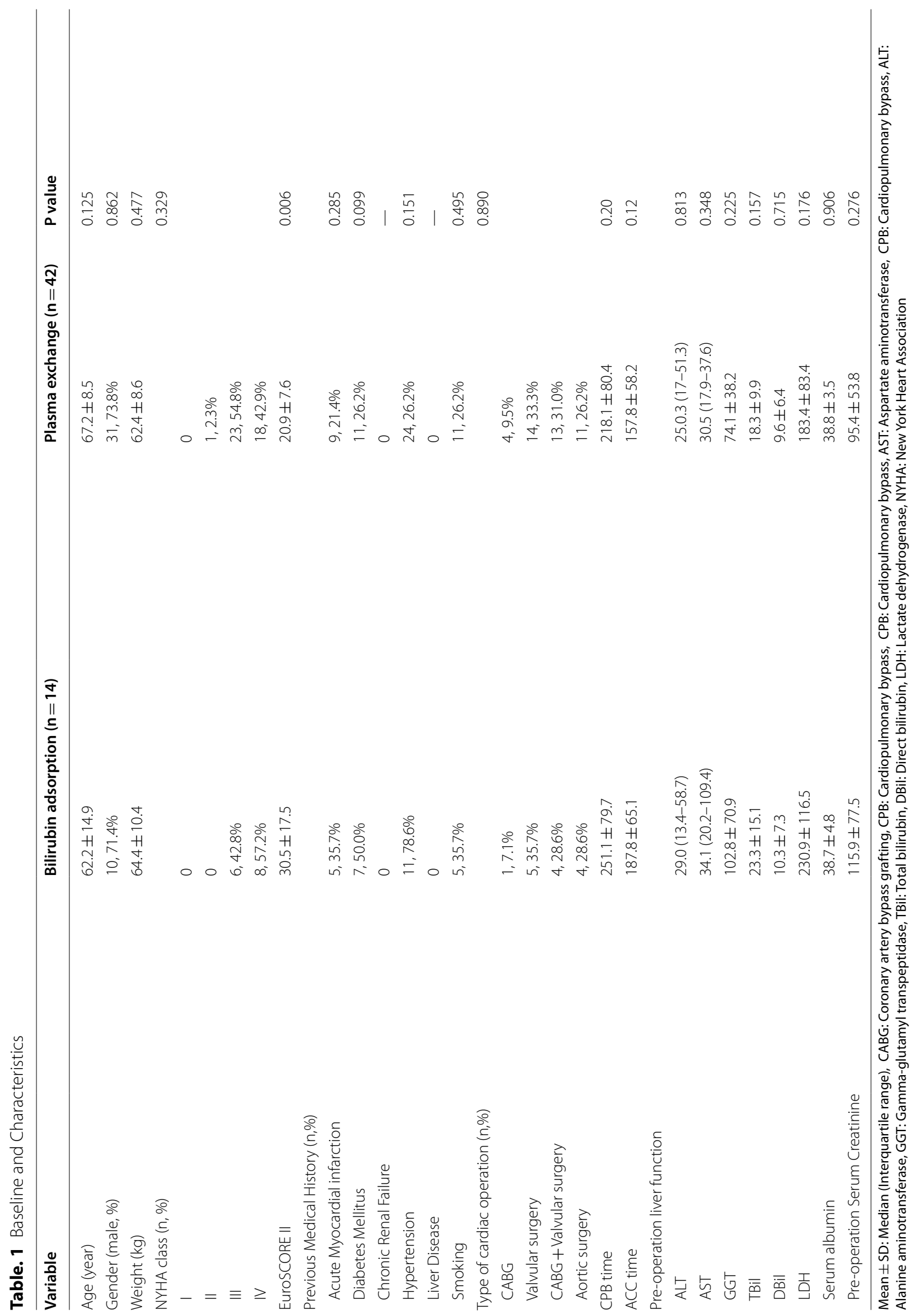




\section{Results}

Preoperative and intraoperative variables are listed in Table 1 . The mean age of all the patients was $66.0 \pm 10.6$ years. Most of the patients were male (41/56, $73.2 \%$ ) and had cardiac insufficiency (NYHA class III/IV, $55 / 56,98.2 \%)$. The patients in the BA treatment group had higher risks of surgery mortality with higher EuroScore II scores than those in the PEX treatment group $(p=0.006)$. None of the patients had a history of chronic renal failure or liver disease. Baseline liver function and renal function were not different between the two groups. The type of cardiac operation showed no differences between the two groups. The mean cardiopulmonary bypass time and aortic cross-clamp were $225.9 \pm 80.7$ and $164.9 \pm 60.7 \mathrm{~min}$, respectively. The BA treatment group presented a longer bypass time and cross-clamp time, while there were no differences between the two groups.

The baseline total bilirubin (TBil) level of all patients before treatment was $306.0 \pm 100.7 \mu \mathrm{mol} / \mathrm{l}$, and the direct bilirubin (DBil) level was 174.0 $\pm 68.3 \mu \mathrm{mol} / \mathrm{l}$. Before adsorption or exchange, DBil $(\mathrm{p}=0.040)$ levels were much higher in the BA treatment group than in the PEX treatment group. Compared to the plasma exchange treatment, the bilirubin adsorption treatment provided a statistically significant reduction in TBil $(p=0.016)$ and DBil $(p=0.036)$ levels. The details of the levels of TBil and DBil before and after BA/PE treatment are shown in Table 2.

Serum aminotransferase levels reflecting hepatocyte cytolysis syndrome also significantly improved: aspartate aminotransferase (AST) and alanine aminotransferase (ALT) levels were both decreased after BA or PEX treatment (Table 2). Since the majority of the patients with AFL received renal replacement therapy before BA or PEX treatment for MODS, the baseline serum creatinine and blood urea nitrogen (BUN) levels were not very serious: the serum creatinine level was $112.3 \pm 43.4 \mu \mathrm{mol} / \mathrm{l}$, and the BUN level was $14.5 \pm 6.0 \mathrm{mmol} / \mathrm{l}$. After BA or PEX treatment, the serum creatinine level was $86.8 \pm 27.8 \mu \mathrm{mol} / \mathrm{l}$, and the BUN level was $12.1 \pm 3.7 \mathrm{mmol} / \mathrm{l}$ (Table 2). The improvement in renal function indicated that both treatments could eliminate water-soluble toxic substances.

The in-hospital mortality was $85.7 \%(48 / 56)$ in the whole group, and the BA group had a trend toward a lower mortality than the PEX group $(71.4 \%$ vs. $90.5 \%$, $\mathrm{P}=0.078)$. BA treatment showed better circulatory support, including lower risks of IABP (21.4\% vs. $52.4 \%$, $\mathrm{p}=0.044)$, ECMO (21.4\% vs. $50.0 \%, \mathrm{p}=0.061)$, reintubation $(64.3 \%$ vs. $40.5 \%, \mathrm{p}=0.122)$ and ventricular arrhythmias $(64.3 \%$ vs. $45.2 \%, \mathrm{p}=0.217)$. One of the main efficacy criteria of treatment therapy was its impact on maintaining homeostasis. BA treatment reduced the incidence of hepatic encephalopathy $(35.7 \%$ vs. $71.4 \%$, $\mathrm{p}=0.017)$ and septic shock (35.7\% vs. $52.4 \%, \mathrm{p}=0.280)$. The peak lac adsorption $(\mathrm{p}=0.004)$ and vasoactive inotropic score (VIS) $(\mathrm{p}=0.002)$ after treatment were both lower in the BA treatment group than in the PEX treatment group. Although the difference in the two groups was not statistically significant, there seems to be some practical difference in that the BA treatment group showed better clinical efficacy and outcomes. The detailed early outcomes are listed in Table 3.

After matching, a total of 28 patients were enrolled in the analysis (14 in the BA treatment group and 14 in the PEX treatment group). The in-hospital mortality was lower in the BA treatment group than in the PEX treatment group $(71.4 \%$ vs. $100 \%, \mathrm{P}=0.049)$. The circulatory supports were similar between the two groups, including the usage of IABP, ECMO, reintubation and ventricular arrhythmias. The BA treatment group had advantages in maintaining homeostasis, reflecting a lower incidence of hepatic encephalopathy $(35.7 \%$ vs. $92.9 \%, \mathrm{p}=0.002)$ and lower VIS score $(\mathrm{P}=0.013)$ and lac levels $(\mathrm{P}=0.045)$ after treatment. The BA treatment group also showed better efficacy in removing toxins and exhibited a statistically significant reduction in TBil, DBil, ALT, AST, serum creatinine, BUN and C-creative protein levels. The results of the matched cohort are listed in Table 4.

\section{Discussion}

The occurrence of hyperbilirubinemia after cardiac surgery has been observed for a long time, and despite demonstrable improvements in surgical techniques and perioperative care over the last decade, hepatic dysfunction remains a serious postoperative complication [10, 11]. Relevant factors for hyperbilirubinemia after cardiac surgery include [4, 12-14] the following: (1) hepatic ecchymosis due to high pressure of the right atrium; (2) nonpulsatile flow in $\mathrm{CPB}$ and its associated risk of regional malperfusion causing liver ischemic damage; (3) massive transfusion; (4) hemolysis caused by cardiotomy suction, membrane oxygenation and various other elements of CPB; and (5) postoperative infection. Different methods have been used to treat hyperbilirubinemia, such as the molecular adsorbent recirculating system (MARS), plasma exchange (PEX) and bilirubin adsorption (BA). It is still unclear which treatment strategy is more useful for patients with hyperbilirubinemia after cardiac surgery15. We presented a retrospective analysis of prospectively collected data that compared BA treatment with PEX treatment in hyperbilirubinemia after cardiac surgery. This study demonstrated the following: (I) BA treatment could be considered an effective strategy for the reduction of TBil and DBil levels in patients with postoperative hyperbilirubinemia. (II) Compared to 


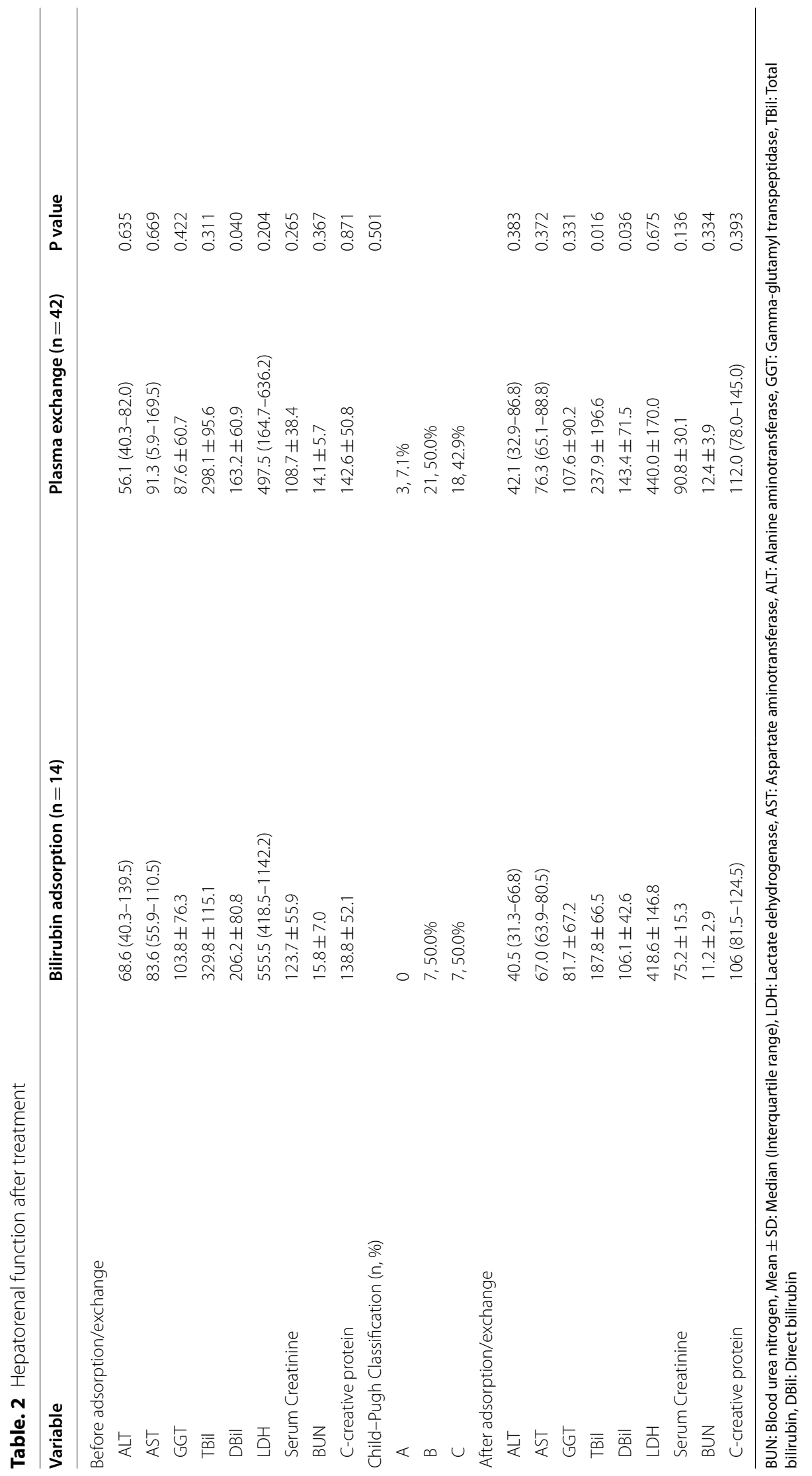


Table. 3 Outcomes

\begin{tabular}{llll}
\hline Variable & Bilirubin adsorption $(\mathbf{n}=\mathbf{1 4})$ & Plasma exchange $(\mathbf{n}=\mathbf{4 2})$ & P value \\
\hline Death $(n, \%)$ & $10,71.4 \%$ & $38,90.4 \%$ & 0.078 \\
CRRT $(n, \%)$ & $12,85.7 \%$ & $30,71.4 \%$ & 0.285 \\
IABP $(n, \%)$ & $3,21.4 \%$ & $22,52.4 \%$ & 0.044 \\
ECMO $(n, \%)$ & $3,21.4 \%$ & $21,50.0 \%$ & 0.061 \\
Re-intubation $(n, \%)$ & $9,64.2 \%$ & $17,40.5 \%$ & 0.122 \\
Septic shock (n, \%) & $5,35.7 \%$ & $22,52.4 \%$ & 0.280 \\
Ventricular arrhythmias (n, \%) & $9,64.2 \%$ & $19,45.2 \%$ & 0.217 \\
Hepatic encephalopathy (n, \%) & $5,35.7 \%$ & $30,71.4 \%$ & 0.017 \\
Peak VIS after adsorption/exchange & $51.5 \pm 12.2$ & $65.1 \pm 15.6$ & 0.002 \\
Peak lac adsorption/exchange (mmol/L) & $8.4 \pm 2.1$ & $10.6 \pm 2.3$ & 0.004 \\
\hline
\end{tabular}

Lac: arterial lactate

Table. 4 Outcomes and hepatorenal function in matched cohort

\begin{tabular}{|c|c|c|c|}
\hline Variable & Bilirubin adsorption $(n=14)$ & Plasma exchange $(n=14)$ & $P$ value \\
\hline Death $(n, \%)$ & $10,71.4 \%$ & $14,100.0 \%$ & 0.049 \\
\hline CRRT $(n, \%)$ & $12,85.7 \%$ & $12,85.7 \%$ & - \\
\hline $\operatorname{IABP}(n, \%)$ & $3,21.4 \%$ & $8,57.15 \%$ & 0.060 \\
\hline $\mathrm{ECMO}(\mathrm{n}, \%)$ & $3,21.4 \%$ & $3,21.4 \%$ & - \\
\hline Re-intubation (n, \%) & $9,64.2 \%$ & $10,71.4 \%$ & 0.500 \\
\hline Septic shock (n, \%) & $5,35.7 \%$ & $4,28.6 \%$ & 0.500 \\
\hline Ventricular arrhythmias (n, \%) & $9,64.2 \%$ & $6,42.9 \%$ & 0.225 \\
\hline Hepatic encephalopathy (n, \%) & $5,35.7 \%$ & $13,92.9 \%$ & 0.002 \\
\hline Peak VIS after adsorption/exchange & $47.3 \pm 5.9$ & $62.9 \pm 19.8$ & 0.013 \\
\hline Peak lac adsorption/exchange (mmol/L) & $8.4 \pm 2.2$ & $10.1 \pm 2.1$ & 0.045 \\
\hline \multicolumn{4}{|l|}{ After adsorption/exchange } \\
\hline ALT & $28.9(19.4-64.0)$ & $42.1(32.9-94.0)$ & 0.021 \\
\hline AST & $58.0(46.6-74.5)$ & $76.3(60.3-101.7)$ & 0.012 \\
\hline GGT & $69.0(20.0-118.5)$ & $79.0(30.0-125.3)$ & 0.667 \\
\hline TBil & $162.9 \pm 76.3$ & $235.2 \pm 82.9$ & 0.024 \\
\hline DBil & $90.4 \pm 35.2$ & $139.1 \pm 53.5$ & 0.009 \\
\hline LDH & $394.5 \pm 127.8$ & $435.1 \pm 161.7$ & 0.467 \\
\hline Serum Creatinine & $80.1 \pm 11.9$ & $107.8 \pm 22.8$ & 0.001 \\
\hline BUN & $10.3 \pm 2.8$ & $14.5 \pm 4.3$ & 0.010 \\
\hline C-creative protein & $83.5 \pm 15.8$ & $151.6 \pm 49.2$ & 0.018 \\
\hline
\end{tabular}

PEX treatment, BA treatment could reduce in-hospital mortality and risks of poor outcomes.

Several studies have reported that the incidence of hyperbilirubinemia after cardiac surgery is between 10 and $40 \%[4,16,17]$, which has been consistent since the first report in 1967 [2]. In our center, the incidence of hyperbilirubinemia is $0.48 \%$, which is similar to previous studies. Most patients suffered severe cardiac disease with NYHA class III/IV [18], and valvular surgery and CABG were the most common surgery types. These findings are similar to those of previous studies, and complicated valve surgery procedures caused more frequent postoperative hyperbilirubinemia [4, 12, 18-20]. Therefore, the severity and complexity of valve surgery might be important predictive factors for the incidence of postoperative hyperbilirubinemia.

When hyperbilirubinemia turned to acute liver failure, a large amount of endotoxin, cytokines and other pathogenic factors, especially those associated with albumin, accumulated in plasma. The combined toxin was difficult to pass through traditional blood purification treatments such as hemodialysis. These toxins play a key role in the development of liver failure and can cause hemodynamics and hepatorenal syndrome. It has been proven that 
short-term mortality depends on high levels of bilirubin [21], and low levels of bilirubin can facilitate hepatocyte regeneration. High levels of bile acids may induce apoptosis and cell necrosis of hepatocytes and retard hepatic regeneration [22]. In addition, bilirubin has neurotoxic and encephalopathic effects [23]. For these reasons, the removal of bilirubin seems to be an important therapeutic target. BA treatment and PEX treatment are both effective therapies for hyperbilirubinemia $[24,25]$. Plasma exchange therapy can remove a variety of toxins, supply coagulation factors and regulate immune function. Bilirubin adsorption works through resin adsorbents, which have acceptable capacity for toxins such as bilirubin and cytokines. In our study, we found that BA treatment had a higher removal ability for TBil and DBil than PEX treatment, while the removal ability of ALT, AST and serum creatinine was similar between the two treatments. The following rate-limiting factors influenced the removal ability of albumin-binding toxins: [1] plasma ion strength and $\mathrm{pH}$ value [26]; [2] the possible loss of albumin due to its binding to the absorber columns [27]; and [3] the molar ratio of bilirubin to albumin [28]. The 20 -fold higher molar ratio of serum bilirubin to albumin compared to the respective dialysate $[26,29]$ and the loss of albumin with time were due to its binding to the filter [27].

Our findings demonstrated that postoperative hyperbilirubinemia resulted in significantly increased in-hospital mortality, as the mortality was up to $85.7 \%$, which was much higher than the reported early mortality between 19 and 25\% [3, 18]. Patients in our study were all critical patients with severe congestive heart disease, and most of them were NYHA class III/IV. EuroSCORE II scores showed that these patients suffered huge risks of mortality and complications. Indeed, patients in our study had almost acute liver failure with MODS, and the mortality of MODS after cardiac surgery was reported to be up to $90 \%[5,6]$. Acute liver failure combined with MODS can cause disorder of the internal environment and hemodynamic instability. In this study, most patients suffered poor clinical outcomes, especially in the usage of IABP, ECMO, CRRT, etc. Almost all the patients died of MODS.

There have been limited studies regarding the optimal techniques for bilirubin removal, and no direct comparison exists between BA and PEX in patients after cardiac surgery. Recently, Chen $\mathrm{X}$ and his colleagues concluded that BA treatment was an effective and safe method for treating hyperbilirubinemia in patients after cardiac surgery [30]. Our study added evidence that BA treatment not only had a higher bilirubin removal ability but also could lower the mortality and risks of poor clinical outcomes in patients with hyperbilirubinemia after cardiac surgery. Moreover, PEX treatment requires a large amount of plasma or albumin, which could be associated with limitations regarding plasma and patients with rare blood types. BA treatment has an advantage in this aspect; it can adsorb bilirubin in a competitive binding manner compared to albumin. After the free bilirubin in plasma is adsorbed, the bilirubin bound to plasma albumin is partially dissociated and then adsorbed, and albumin and coagulation factors are protected in this way.

\section{Study limitations}

This study was a retrospective study with limited patients in one center. Missing data for other possible factors, such as the amount of bleeding, postoperative central venous pressure and coagulation function, may limit our findings. We tried to compare two treatment therapies more clearly according to a matched cohort. However, factors that affect assignment to treatment and outcomes but that cannot be observed could not have been accounted for in the matched cohort. Moreover, after matching, the sample size was decreased, which may result in some statistical errors. Furthermore, the patients in the two groups were chosen from different periods, which may also cause some potential errors. These hidden biases might have remained in the matched cohort and led to statistical errors. In addition, the treatment timing for different therapies was not discussed in this study, and we thought a larger sample size and prospective studies would be needed to investigate this issue.

\section{Conclusion}

Compared to PEX treatment, BA treatment had a higher bilirubin removal ability in patients with hyperbilirubinemia and could reduce the mortality and risks of poor clinical outcomes. BA treatment should be considered an effective treatment method for patients with higher TBil levels or DBil levels.

\section{Abbreviations \\ CABG: Coronary artery bypass grafting; CPB: Cardiopulmonary bypass; ACC: Aortic cross-clamp; AST: Aspartate aminotransferase; ALT: Alanine aminotrans- ferase; GGT: Gamma-glutamyl transpeptidase; TBil: Total bilirubin; DBil: Direct bilirubin; LDH: Lactate dehydrogenase; NYHA: New York Heart Association; BUN: Blood urea nitrogen; Lac: Arterial lactate; CRRT: Continuous renal replacement therapy; IABP: Intra-aortic balloon pump; ECMO: Extracorporeal membrane oxygenation.}

\section{Authors' contributions}

Dong-jin Wang and Hai-long Cao were major contributors to the conception of the study and revised the manuscript; Ke Pan, He Zhang, Kai Zhong, Hai-tao Zhang, Zhong Chen, Ze-shi Li, Man Xie, Su-ping Gu and Tuo Pan collected the data; Ke Pan and He Zhang wrote the manuscript; all the authors contributed to the conception of the study; Tuo Pan, Ke Pan and He Zhang corrected the statistical analysis. All the authors read and approved the final manuscript. 


\section{Funding}

This work has been supported by the National Key R\&D program of China (2016YFC1000808), National Natural Science Foundation of China (No. 81970401, No.81670437) and Jiangsu Provincial Key Medical Discipline (ZDXKA2016019).

\section{Availability of data and materials}

The datasets used and/or analyzed during the current study are available from the corresponding author on reasonable request.

\section{Declarations}

\section{Ethics approval and consent to participate}

Ethical approval was obtained from the Medical Ethics Committee of Affiliated Nanjing Drum Tower Hospital, Nanjing University Medical College (2020-249$01)$, and the study was conducted according to the principles in the Declaration of Helsinki.

\section{Consent for publication}

All the authors have read and approved the manuscript for publication.

\section{Competing interests}

The authors declare that they have no competing interests.

\section{Author details}

${ }^{1}$ Department of Cardio-Thoracic Surgery, Nanjing Drum Tower Clinical College of Xuzhou Medical University, Xuzhou, China. ${ }^{2}$ Department of Cardio Thoracic Surgery, Nanjing Drum Tower Hospital, Chinese Academyof Medical Science and Peking Union Medical College, Graduate School of Peking UnionMedical College, Beijing, China. ${ }^{3}$ Department of Cardio-Thoracic Surgery, Nanjing Drum Tower Hospital Clinical College of Nanjing Medical University, Number 321 Zhongshan Road, Jiangsu 210008, China. ${ }^{4}$ Department of Cardio-Thoracic Surgery, Nanjing Drum Tower Hospital, The Affiliated Hospital of Nanjing University Medical School, Number 321 Zhongshan Road, Jiangsu 210008, China.

Received: 15 April 2021 Accepted: 12 Auqust 2021

Published online: 23 August 2021

\section{References}

1. Welbourn N, Melrose DG, Moss DW. Changes in serum enzyme levels accompanying cardiac surgery with extracorporeal circulation. J Clin Pathol. 1966:19(3):220-32.

2. Lockey E, McIntyre N, Ross DN, Brookes E, Sturridge MF. Early jaundice after open-heart surgery. Thorax. 1967;22(2):165-9.

3. Collins JD, Bassendine MF, Ferner R, Blesovsky A, Murray A, Pearson DT, et al. Incidence and prognostic importance of jaundice after cardiopulmonary bypass surgery. Lancet. 1983;1(8334):1119-23.

4. Wang MJ, Chao A, Huang CH, Tsai CH, Lin FY, Wang SS, et al. Hyperbilirubinemia after cardiac operation. Incidence, risk factors, and clinical significance. J Thorac Cardiovasc Surg. 1994;108(3):429-36.

5. Daas M, Plevak DJ, Wijdicks EF, Rakela J, Wiesner RH, Piepgras DG, et al. Acute liver failure: results of a 5-year clinical protocol. Liver Transpl Surg. 1995;1 (4):210-9.

6. Gerth HU, Pohlen M, Pavenstadt H, Schmidt H. Extracorporeal liver support of liver failure. Z Gastroenterol. 2017:55(4):383-93.

7. O'Grady JG, Schalm SW, Williams R. Acute liver failure: redefining the syndromes. Lancet. 1993;342(8866):273-5.

8. Bernal W, Wendon J. Acute liver failure. N Engl J Med. 2013:369(26):2525-34.

9. Jalan R, Yurdaydin C, Bajaj JS, Acharya SK, Arroyo V, Lin HC, et al. Toward an improved definition of acute-on-chronic liver failure. Gastroenterology. 2014;147(1):4-10.

10. Olsson R, Hermodsson S, Roberts D, Waldenstrom J. Hepatic dysfunction after open-heart surgery. Scand J Thorac Cardiovasc Surg. 1984;18(3):217-22.
11. Michalopoulos A, Alivizatos P, Geroulanos S. Hepatic dysfunction following cardiac surgery: determinants and consequences. Hepatogastroenterology. 1997;44(15):779-83.

12. Hosotsubo KK, Nishimura M, Nishimura S. Hyperbilirubinaemia after major thoracic surgery: comparison between open-heart surgery and oesophagectomy. Crit Care. 2000;4(3):180-7.

13. Gregoretti S. Suction-induced hemolysis at various vacuum pressures: implications for intraoperative blood salvage. Transfusion. 1996:36(1):57-60.

14. Garcia MJ, Vandervoort P, Stewart WJ, Lytle BW, Cosgrove DM, 3rd, Thomas JD, et al. Mechanisms of hemolysis with mitral prosthetic regurgitation. Study using transesophageal echocardiography and fluid dynamic simulation. J Am Coll Cardiol. 1996;27(2):399-406.

15. Szczepiorkowski ZM, Shaz BH, Bandarenko N, Winters JL. The new approach to assignment of ASFA categories-introduction to the fourth special issue: clinical applications of therapeutic apheresis. J Clin Apher. 2007;22(3):96-105.

16. Scott BB, McCormack MC, Austen WG. Commentary on: Compact Fat Grafting: A Novel Method to Improve Graft Retention Through Modulation of Adipocyte Size. Aesthet Surg J. 2021.

17. Chu CM, Chang CH, Liaw YF, Hsieh MJ. Jaundice after open heart surgery: a prospective study. Thorax. 1984;39(1):52-6.

18. Nishi H, Sakaguchi T, Miyagawa S, Yoshikawa Y, Fukushima S, Saito S, et al. Frequency, risk factors and prognosis of postoperative hyperbilirubinemia after heart valve surgery. Cardiology. 2012;122(1):12-9.

19. Lau GT, Tan HC, Kritharides L. Type of liver dysfunction in heart failure and its relation to the severity of tricuspid regurgitation. Am J Cardiol. 2002;90(12):1405-9.

20. Mastoraki A, Karatzis E, Mastoraki S, Kriaras I, Sfirakis P, Geroulanos S. Postoperative jaundice after cardiac surgery. Hepatobiliary Pancreat Dis Int. 2007:6(4):383-7.

21. Lopez-Velazquez JA, Chavez-Tapia NC, Ponciano-Rodriguez G, SanchezValle V, Caldwell SH, Uribe M, et al. Bilirubin alone as a biomarker for shortterm mortality in acute-on-chronic liver failure: an important prognostic indicator. Ann Hepatol. 2013;13(1):98-104.

22. Chamuleau RA, Aronson DC, Frederiks WM, Bosman DK, Smit JJ, Maas MA et al. Liver regeneration after partial hepatectomy in rats with defective bilirubin conjugation or biliary excretion. Dig Dis Sci. 1991;36(4):510-2.

23. Lee JY, Kim SB, Chang JW, Park SK, Kwon SW, Song KW, et al. Comparison of the molecular adsorbent recirculating system and plasmapheresis for patients with graft dysfunction after liver transplantation. Transplant Proc. 2010:42(7):2625-30.

24. Onodera K, Sakata H, Yonekawa M, Kawamura A. Artificial liver support at present and in the future. J Artif Organs. 2006;9(1):17-28.

25. Li LJ, Yang Q, Huang JR, Xu XW, Chen YM, Fu SZ. Effect of artificial liver support system on patients with severe viral hepatitis: a study of four hundred cases. World J Gastroenterol. 2004;10(20):2984-8.

26. Gong D, Cruz D, Ronco C. Depurative capacity of molecular adsorbent recycling system (MARS): A focus on bilirubin removal. Int J Artif Organs. 2008;31(10):875-81.

27. Gong D, Ji D, Ren B, Tao J, Xu B, Ronco C, et al. Significant decrease in dialysate albumin concentration during molecular adsorbent recirculating system (M.A.R.S.) therapy. Int J Artif Organs. 2008:31(4):333-9.

28. Steiner C, Sen S, Stange J, Williams R, Jalan R. Binding of bilirubin and bromosulphthalein to albumin: implications for understanding the pathophysiology of liver failure and its management. Liver Transpl. 2004;10(12):1531-8.

29. Schaefer B, Schaefer F, Engelmann G, Meyburg J, Heckert KH, Zorn M, et al. Comparison of Molecular Adsorbents Recirculating System (MARS) dialysis with combined plasma exchange and haemodialysis in children with acute liver failure. Nephrol Dial Transplant. 2011;26(11):3633-9.

30. Chen X, Li L, Bai M, Sun S, Chen X. Bilirubin adsorption for the treatment of severe hyperbilirubinemia after cardiac surgery: A retrospective cohort study. Int J Artif Organs. 2021:391398821997841.

\section{Publisher's Note}

Springer Nature remains neutral with regard to jurisdictional claims in published maps and institutional affiliations. 